\title{
$247 \mathrm{~nm}$ solar-blind ultraviolet $\boldsymbol{p}$ - $\boldsymbol{i}-\boldsymbol{n}$ photodetector
}

\author{
V. V. Kuryatkov, B. A. Borisov, and S. A. Nikishin ${ }^{\text {a) }}$ \\ Department of Electrical and Computer Engineering, Texas Tech University, Lubbock, Texas 79409 \\ Yu. Kudryavtsev and R. Asomoza \\ SIMS Laboratory of SEES, Department of Electrical Engineering, CINVESTAV, \\ Mexico Distrito Federal 07300, Mexico \\ V. I. Kuchinskii and G. S. Sokolovskii \\ Ioffe Physico-Technical Institute, 26 Polytechnicheskaya, St. Petersburg 194021, \\ Russian Federation \\ D. Y. Song and M. Holtz \\ Department of Physics, Texas Tech University, Lubbock, Texas 79409
}

(Received 8 May 2006; accepted 24 August 2006; published online 6 November 2006)

\begin{abstract}
We describe solar-blind $p-i-n$ photodetectors based on short period superlattices of $\mathrm{AlN} / \mathrm{Al}_{0.08} \mathrm{Ga}_{0.92} \mathrm{~N}$. The devices are grown using gas source molecular beam epitaxy with ammonia on transparent sapphire substrates. The cutoff wavelength for the device is $247 \mathrm{~nm}$. For diodes with $150 \mu \mathrm{m}$ diameter mesas and sidewall surfaces passivated in oxygen plasma, we obtain extremely low dark leakage current of $\sim 3 \mathrm{pA} / \mathrm{cm}^{2}$ and high zero-bias resistance of $\sim 6 \times 10^{14} \Omega$. At $10 \mathrm{~V}$ reverse bias the observed responsivity is $62 \mathrm{~mA} / \mathrm{W}$. (C) 2006 American Institute of Physics. [DOI: $10.1063 / 1.2364049$ ]
\end{abstract}

AlGaN-based solar-blind photodetectors (PDs) have been demonstrated recently. ${ }^{1-3}$ Device designs with response in the $240-290 \mathrm{~nm}(5.17-4.28 \mathrm{eV})$ range require $n$-type, intrinsic, and $p$-type $\mathrm{AlGaN}$ with AlN mole fractions of 0.5 and higher. High performance $p-i-n$ PD structures designed for back illumination must be grown on UV transparent substrates. The $i$ region must absorb light in the desired wavelength range and be sandwiched by wider band gap (transparent) $n$ - and $p$-type cladding regions.

PDs based on AlGaInN alloys have been grown primarily by metal organic chemical vapor deposition (MOCVD). ${ }^{4,5}$ However, forming high quality AlGaN $p-i-n$ structures which exhibit low leakage current and desirable $p$-type doping level is difficult at high AlN mole fraction. One major problem is the high defect density due to substrate-lattice mismatch. The second problem is the large acceptor activation energy of $p$-type dopant $\mathrm{Mg}$ in high $\mathrm{Al}$ content alloys. We have shown that short period superlattices (SPSLs) of $\mathrm{AlN} / \mathrm{Al}_{0.08} \mathrm{Ga}_{0.92} \mathrm{~N}$ can be grown using gas source molecular beam epitaxy (GSMBE) to achieve both $n$ and $p$-type materials with large optical gaps suitable for deep UV optoelectronic devices. ${ }^{6-8}$

We describe here the use of SPSLs for preparing solarblind $p-i-n$ PDs and describe their characteristics. The PDs exhibit low dark leakage current density of $\sim 3 \mathrm{pA} / \mathrm{cm}^{2}$, high zero-bias resistance of $\sim 6 \times 10^{14} \Omega$, and peak responsivity of $62 \mathrm{~mA} / \mathrm{W}$ at $247 \mathrm{~nm}$ under $10 \mathrm{~V}$ reverse bias.

The average composition and the resulting effective energy gap of AlN/AlGaN SPSL depends on the well/barrier thickness ratio, the well composition, and the SL period. Effective band gaps between $4.5 \mathrm{eV}(276 \mathrm{~nm})$ and $5.3 \mathrm{eV}$

\footnotetext{
a) Author to whom correspondence should be addressed; electronic mail: sergey.a.nikishin@ttu.edu
}

$(234 \mathrm{~nm})$, as determined by optical reflectivity measurements, ${ }^{9}$ were obtained. Here, in order to compare the optical properties of our SPSLs with AlGaN random alloys, reflectance and transmittance were measured between 200 and $900 \mathrm{~nm}$. Test SPSLs and AlGaN layers of comparable average AlN content $(\sim 70 \%)$ and total thickness $(\sim 400 \mathrm{~nm})$ were grown using identical buffer layers ( $\sim 40 \mathrm{~nm}$ thick AlN on sapphire) and without intentional doping. Using the method of Poruba et al. ${ }^{10}$ the reflectance and transmittance spectra were used to obtain the absorption coefficient $(\alpha)$ and index of refraction. Self-consistent expressions are numerically solved to obtain $\alpha$ at each photon energy $(\hbar \omega)$. For transitions across direct band gap semiconductors, the absorption depends on photon energy according to

$$
\alpha(\hbar \omega) \propto \sqrt{\hbar \omega-E_{g}}
$$

just above the optical gap $E_{g}$. In Fig. 1 we graph $\alpha^{2}$ versus photon energy, which is expected to rise linearly above $E_{g}$, for an AlGaN alloy layer. We note that the shapes of the alloy and superlattice absorption edges are comparable when the composition shift of $E_{g}$ is taken into account. The magnitudes of the $\alpha^{2}$ values are also consistent. For reference, Fig. 1 (inset) shows raw transmittance data for the same samples. These spectra are likewise comparable. A linear fit of $\alpha^{2}$ versus photon energy data to $\alpha^{2}=0$ is used to estimate $E_{g}$. The conclusion from these studies is that SPSLs with extremely short period have optical absorption properties comparable to AlGaN random alloys, allowing us to apply similar design concepts for UV photodetectors.

Device structures were grown on 2 in. (0001) sapphire substrates using GSMBE with ammonia. ${ }^{11}$ The growth started with an AlN nucleation layer, followed by an undoped $\sim 300 \mathrm{~nm}$ thick SPSL with an average $x_{\mathrm{AIN}}$ content of 


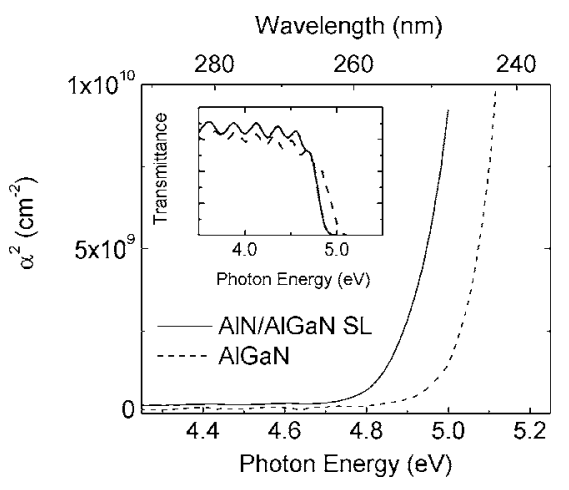

FIG. 1. Optical absorption spectra of $\mathrm{AlN} / \mathrm{Al}_{0.08} \mathrm{Ga}_{0.92} \mathrm{~N}$ SPSL and random AlGaN alloy with similar average content of AlN and effective optical band gap. Inset shows the results of transmittance measurements for the same samples.

0.7 and transparent to $240 \mathrm{~nm}$. These AlN and SPSL are required to mitigate dislocation density in the subsequent active layer of the device. ${ }^{8,12,13}$ The $n$-, $i$-, and $p$-type layers are composed of $\mathrm{AlN} / \mathrm{Al}_{0.08} \mathrm{Ga}_{0.92} \mathrm{~N}$ SPSLs having different optical gaps and thicknesses. These layers were grown in the two-dimensional (2D) growth mode. The SPSL growth rate was $\sim 300 \mathrm{~nm} / \mathrm{h}(0.33 \mathrm{ML} / \mathrm{s})$ to ensure the fine control of the barrier and well thicknesses necessary to obtain optical band gaps from $\sim 4.96 \mathrm{eV}(\sim 250 \mathrm{~nm})$ in $i$ layer to $\sim 5.17 \mathrm{eV}(\sim 240 \mathrm{~nm})$ in $n$ - and $p$-type layers. The thicknesses of $n, i$, and $p$ layers were varied in the range from $200 \mathrm{~nm}$ to $350 \mathrm{~nm}$, from 40 to $120 \mathrm{~nm}$, and from 100 to $250 \mathrm{~nm}$, respectively. The SPSLs were either not intentionally doped ( $i$ type) or doped with $\mathrm{Si}$ derived from silane ( $n$ type), or $\mathrm{Mg}$ derived from effusion cell ( $p$ type).

A typical distribution of $\mathrm{Si}$ and $\mathrm{Mg}$ and SPSL optical band gaps through $p-i-n$ part of photodetector structure are shown in Fig. 2. Using the dependences of $E_{g}$ on SPSL period with 2 and $3 \mathrm{ML}$ (monolayer) thick $\mathrm{Al}_{0.08} \mathrm{Ga}_{0.92} \mathrm{~N}$ wells, reported in Refs. 8 and 9, one could estimate the period and well/barrier thicknesses of these SPSLs, namely $\{\sim 9 \mathrm{ML}$ $(\sim 2.25 \mathrm{~nm})$ and $\sim 3 \mathrm{ML}(\sim 0.75 \mathrm{~nm}) / \sim 6 \mathrm{ML}(\sim 1.5 \mathrm{~nm})\}$ and $\{\sim 8 \mathrm{ML}(\sim 2 \mathrm{~nm})$ and $\sim 2 \mathrm{ML}(\sim 0.50 \mathrm{~nm}) / \sim 6 \mathrm{ML}$ $(\sim 1.5 \mathrm{~nm})\}$ for $i$ and $p(n)$ regions, respectively. The average concentrations of $\mathrm{Si}$ and $\mathrm{Mg}$ measured by secondary ion mass spectrometry (SIMS) were $\sim 7 \times 10^{19}$ and $\sim 1$ $\times 10^{20} \mathrm{~cm}^{-3}$, respectively. Capacitance-voltage $(C-V)$ measurements of the structure for which we show SIMS data in

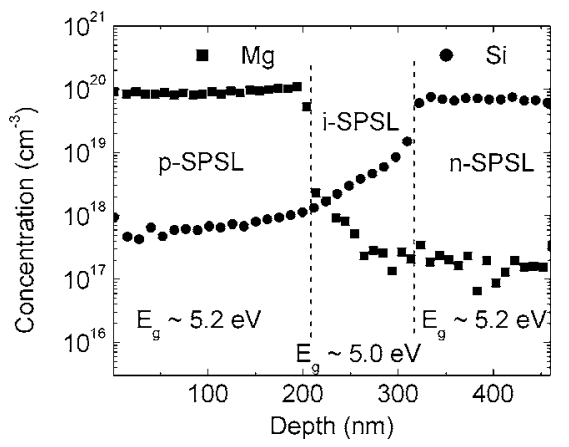

FIG. 2. SIMS profiles of $\mathrm{Mg}$ and $\mathrm{Si}$ for the $p-i-n$ region of the double heterostructure $247 \mathrm{~nm}$ photodetector. Optical band gaps, $E_{g}$, of each region of device are shown.

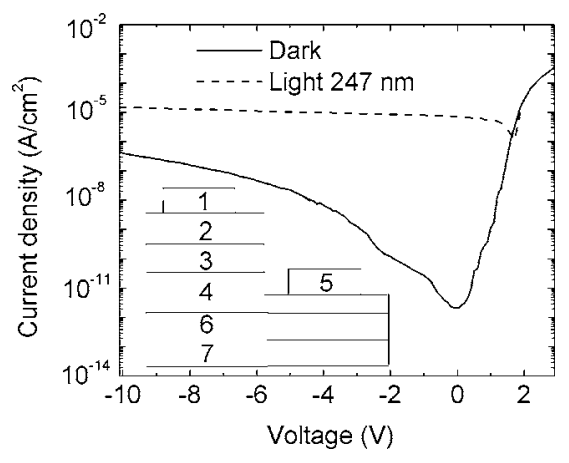

FIG. 3. Current-voltage characteristics of a photodiode in the dark (solid line) and under monochromatic $247 \mathrm{~nm}$ illumination (dashed line). Inset shows the schematic cross section of $\mathrm{PD}$, where $1-\mathrm{Ni} / \mathrm{Au}$ contact, $2-p$ -SPSL, 3- $i$-SPSL, 4- $n$-SPSL, 5-Ti/Al/Ti/Au contact, 6-defect blocking SPSL, and 7-AlN buffer on sapphire substrate.

Fig. 2 reveal the width of the depletion region to be $\sim 150 \mathrm{~nm}$ at zero bias. This result is consistent with $\sim 110 \mathrm{~nm} i$-region thickness shown by the vertical dashed lines in Fig. 2. The SIMS data illustrate substantial diffusion of the Si through the $i$ region extending into the $p$-type layer. Although the impurity concentrations of $\mathrm{Mg}$ and $\mathrm{Si}$ in the $p$-type region differ by more than two orders of magnitude, activation of $\mathrm{Si}$ donors is more efficient than $\mathrm{Mg}$ acceptors. This could be one of the reasons for the high series resistance observed under forward bias for our $p-i-n$ structure. The resulting nonuniform electric field across the $i$ region could result in a diminished internal quantum efficiency of the PD.

In parallel experiments we studied the electrical properties of Si-doped $\left(N_{D} \sim 7 \times 10^{19} \mathrm{~cm}^{-3}\right)$ and Mg-doped $\left(N_{A}\right.$ $\sim 1 \times 10^{20} \mathrm{~cm}^{-3}$ ) SPSLs with optical band gap of $\sim 5.2 \mathrm{eV}$. Room temperature Hall measurements yielded an electron concentration $n \sim(3-5) \times 10^{19} \mathrm{~cm}^{-3}$ and mobility of $\sim 30 \mathrm{~cm}^{2} / \mathrm{V} \mathrm{s}$ for $n$-type layers and a hole concentration $p$ $\sim(6-8) \times 10^{17} \mathrm{~cm}^{-3}$ and mobilities of $3-6 \mathrm{~cm}^{2} / \mathrm{V} \mathrm{s}$ for $p$-type material. These results are consistent with a recent report on highly doped AlN/GaN SLs. ${ }^{14}$

Mesa photodiodes were prepared using standard processing. The $150 \mu \mathrm{m}$ diameter mesas were plasma etched with $\mathrm{Cl}_{2}$ using $100 \mathrm{~nm}$ thick $\mathrm{Ni}$ hard masks. ${ }^{15,16} p$ - and $n$-type contacts were processed separately after removal of the $\mathrm{Ni}$ mask. $n$-type contacts were made using $\mathrm{Ti} / \mathrm{Al} / \mathrm{Ti} / \mathrm{Au}$ with the total thickness of $\sim 200 \mathrm{~nm}$. Contacts to $p$-type SPSL were made using $50 \mathrm{~nm}$ of $\mathrm{Ni}$ followed by $70 \mathrm{~nm}$ of Au.

A representative current-voltage $(I-V)$ characteristic of our PD structure is plotted in Fig. 3. Very low dark leakage current of $\sim 0.5 \mathrm{fA}$ is measured at near zero bias. This corresponds to the leakage current density of $\sim 3 \mathrm{pA} / \mathrm{cm}^{2}$ for the $150 \mu \mathrm{m}$ diameter mesa device. The reverse dark current remains below $0.5 \mu \mathrm{A} / \mathrm{cm}^{2}$ up to a bias of $-10 \mathrm{~V}$ and scales with the device area demonstrating the absence of significant contributions to leakage current from the device mesa sidewalls. ${ }^{2}$ These extremely low values of dark current are comparable to the best results demonstrated for AlGaN solarblind PDs grown by MOCVD. ${ }^{1-5}$ The dark current at lower bias, up to $-1.5 \mathrm{~V}$, does not significantly depend on applied voltage and exhibits a slope of $\sim 1-1.3$ in the log-log scale. We believe this low field leakage is due to tunneling of elec- 


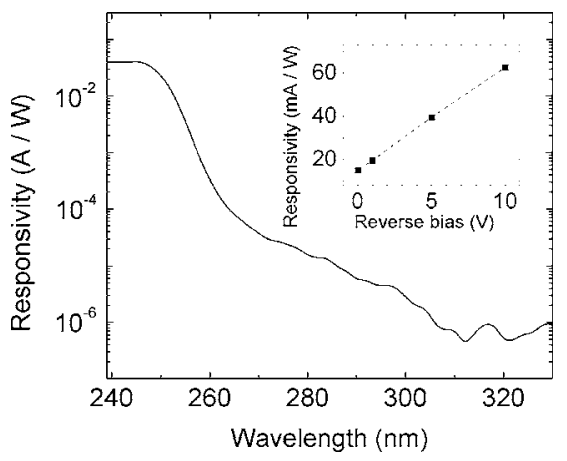

FIG. 4. Spectral responsivity of photodiode measured at $-5 \mathrm{~V}$ reverse bias (bottom illumination). Inset shows responsivity vs reverse bias (247 nm illumination).

trons (holes) from the valence (conduction) band to the conduction (valence) band. The tunneling current is expected to depend on internal electric fields. Recent calculations ${ }^{17}$ have shown that the macroscopic polarization at heterojunction interfaces of AlN/AlGaN SPSLs can be controlled, consistent with low leakage currents obtained in our devices.

At reverse bias over $-3 \mathrm{~V}$ the dark current increases with exponent ranging, in different devices and wafers, from 3 to 6 in the log-log scale. Field-assisted thermal ionization of carriers from traps in the depletion region or combination of hopping conductivity and Poole-Frenkel effect are likely mechanisms for this conductivity. ${ }^{18}$ The range of slopes is typical of AlGaN PDs at high reverse bias, ${ }^{1-6,13}$ and we believe the reverse current correlates with high defect density in structures grown on sapphire. The average density of threading dislocations in our samples, estimated from $\mathrm{x}$-ray measurements, is $\sim 10^{9} \mathrm{~cm}^{-2}$. This is comparable to that reported for AlGaN grown by MOCVD over thin AlN buffer on sapphire. ${ }^{1-3}$

From $(d V / d I)$ we calculate zero-bias PD resistance of $\sim 6 \times 10^{14} \Omega$, resulting in the area-resistance product $R_{0} A$ $\sim 1.2 \times 10^{11} \Omega \mathrm{cm}^{2}$. The PD exhibits excellent monochromatic photoresponse at $247 \mathrm{~nm}$, with $I_{247 \mathrm{~nm}} / I_{\text {dark }} \sim 3 \times 10^{6}$ at zero bias, as shown in Fig. 3 .

The spectral responsivity of PDs under reverse bias is shown in Fig. 4. The peak response was obtained at a wavelength of $\sim 247 \mathrm{~nm}$, in excellent agreement with the expected optical band gap of $\sim 5 \mathrm{eV}$ for the $i$ region of the PD. The device shows excellent rejection of visible radiation. Cutoff at $247 \mathrm{~nm}$ is obtained for these devices, with three orders of magnitude decrease in responsivity from 247 to $265 \mathrm{~nm}$ and four orders of magnitude rejection by $315 \mathrm{~nm}$. This compares well with the rejection ratios in stateof-the-art $p-i-n$ PDs with cutoff at longer wavelength. ${ }^{1-5}$ The substructure visible in the photoresponse in the range of $\sim 265-315 \mathrm{~nm}$ is not understood at this time. It may be related to broadening of the absorption edge due to partial monolayer thickness fluctuations in the well and barrier thickness and formation of the well/barrier interfacial layers with intermediate AlN content. Furthermore, there is little information regarding the effect of intentional doping on the optical absorption properties of AlGaN, which may result in an Urbach tail and affect the device efficiency and spectral response. A study of the temperature dependence of the PD spectral response is needed to examine this hypothesis.

At zero bias the responsivity of $16 \mathrm{~mA} / \mathrm{W}$ was measured at a wavelength of $247 \mathrm{~nm}$. This corresponds to an external quantum efficiency of $\sim 12.5 \%$. The responsivity linearly increases with reverse bias, as shown in the inset of Fig. 4, and reaches its maximum value of $62 \mathrm{~mA} / \mathrm{W}$ at $-10 \mathrm{~V}$. This corresponds to an external quantum efficiency of $30 \%$. This is less than the highest responsivity reported in solar-blind $p-i-n$ PD of $\sim 72 \%$ at $282 \mathrm{~nm}$ Ref. 13 grown by MOCVD. Optimizing the dopant distribution throughout the device, but particularly in the $i$ region, should improve the external quantum efficiency of our PDs. Nevertheless, using the $R_{0} A$ determined above we calculate the thermal noise limited specific detectivity, at zero bias, of $D^{*}=4.5$ $\times 10^{13} \mathrm{~cm} \mathrm{~Hz}^{1 / 2} \mathrm{~W}^{-1}$.

In summary, we have prepared solar-blind heterostructure $p-i-n$ photodetectors based on short period superlattices. Excellent electrical characteristics of $p-n$ junctions formed in these SPSLs demonstrate their potential in solar-blind avalanche photodetectors.

The authors would like to thank Professor H. Temkin for important discussions. Work at Texas Tech University is supported by NSF (ECS-0323640 and ECS-0304224) and the J. F. Maddox Foundation.

${ }^{1}$ R. D. Dupuis and J. C. Campbell, in Wide Energy Bandgap Electronic Devices, edited by F. Ren and J. Zolper (World Scientific, Singapore, 2003), and references therein.

${ }^{2} \mathrm{H}$. Temkin, in Advanced Semiconductor and Organic Nano-techniques Part I, edited by H. Morkoç (Academic, San Diego, 2003), and references therein.

${ }^{3}$ E. Monroy, F. Omnès, and F. Calle, Semicond. Sci. Technol. 18, R33 (2003), and references therein.

${ }^{4}$ E. Ozbay, N. Biyikli, I. Kimukin, T. Kartaloglu, T. Tut, and O. Aytür, IEEE J. Sel. Top. Quantum Electron. 10, 742 (2004), and references therein.

${ }^{5}$ M. B. Reine et al., Proc. SPIE 6121, 237 (2006).

${ }^{6}$ V. Kuryatkov, A. Chandolu, B. Borisov, G. Kipshidze, K. Zhu, S. Nikishin, H. Temkin, and M. Holtz, Appl. Phys. Lett. 82, 1323 (2003).

${ }^{7}$ S. A. Nikishin, V. V. Kuryatkov, A. Chandolu, B. A. Borisov, G. D. Kipshidze, I. Ahmad, M. Holtz, and H. Temkin, Jpn. J. Appl. Phys., Part 2 42, L1362 (2003).

${ }^{8}$ S. A. Nikishin, M. Holtz, and H. Temkin, Jpn. J. Appl. Phys., Part 1 44, 7221 (2005).

${ }^{9}$ M. Holtz, I. Ahmad, V. V. Kuryatkov, B. A. Borisov, G. D. Kipshidze, A. Chandolu, S. A. Nikishin, and H. Temkin, Mater. Res. Soc. Symp. Proc. 798, Y1.9.1 (2004).

${ }^{10}$ A. Poruba et al., J. Appl. Phys. 88, 148 (2000).

${ }^{11}$ G. Kipshidze, V. Kuryatkov, B. Borisov, S. Nikishin, M. Holtz, S. N. G. Chu, and H. Temkin, Phys. Status Solidi A 192, 286 (2002).

${ }^{12}$ H. Wang, J. Zhang, C. Chen, Q. Fareed, J. Yang, and A. Khan, Appl. Phys. Lett. 81, 604 (2002).

${ }^{13}$ R. McClintock, A. Yasan, K. Mayes, D. Shiell, S. R. Darvish, P. Kung, and M. Razeghi, Appl. Phys. Lett. 84, 1248 (2004).

${ }^{14}$ S. Yamaguchi et al., Appl. Phys. Lett. 80, 802 (2002).

${ }^{15}$ V. V. Kuryatkov, G. D. Kipshidze, S. A. Nikishin, P. W. Deelman, and H. Temkin, Phys. Status Solidi A 188, 317 (2001).

${ }^{16}$ K. Zhu, V. Kuryatkov, B. Borisov, G. Kipshidze, S. A. Nikishin, H. Temkin, and M. Holtz, Appl. Phys. Lett. 81, 4688 (2002).

${ }^{17}$ K. A. Bulashevich and S. Yu. Karpov, Phys. Status Solidi C 2, 2394 (2005).

${ }^{18}$ D. V. Kuksenkov, H. Temkin, A. Osinsky, R. Gaska, and M. A. Khan, J. Appl. Phys. 83, 2142 (1998). 